\title{
On the Application of the Multiple Scales Method on Electrostatically Actuated Resonators
}

\author{
Saad Ilyas, Feras K. Alfosail, Mohammad I. Younis* \\ Physical Sciences and Engineering (PSE), King Abdullah University of Science and \\ Technology, 23955-6900, Thuwal, KSA, \\ saad.ilyas@kaust.edu.sa, feras.alfosail@kaust.edu.sa, mohammad.younis@kaust.edu.sa \\ *corresponding author
}

\begin{abstract}
We investigate modeling the dynamics of an electrostatically actuated resonator using the perturbation method of Multiple Time Scales (MTS). First, we discuss two approaches to treat the nonlinear parallel-plate electrostatic force in the equation of motion and their impact on the application of MTS: expanding the force in Taylor series and multiplying both sides of the equation with the denominator of the forcing term. Considering a spring-mass-damper system excited electrostatically near primary resonance, it is concluded that, with consistent truncation of higher-order terms, both techniques yield same modulation equations. Then we consider the problem of an electrostatically actuated resonator under simultaneous superharmonic and primary resonance excitation and derive a comprehensive analytical solution using MTS. The results of the analytical solution are compared against the numerical results obtained by longtime integration of the equation of motion. It is demonstrated that along with the direct excitation components at the excitation frequency and twice of that, higher-order parametric terms should
\end{abstract}


also be included. Finally, the contributions of primary and superharmonic resonance towards the overall response of the resonator are examined.

Keywords: electrostatic force, MEMS resonator, method of Multiple Time Scales, Taylor series expansion, primary resonance excitation, superharmonic resonance excitation

\section{Introduction}

Theoretical and experimental investigations of the nonlinear dynamics of electrostatically actuated micro/nano electromechanical (MEMS/NEMS) beam resonators have been an active area of research since the start of these technologies [1]. MEMS resonators have been used in various applications including sensors [2], mechanical computing [3,4], and radio frequency (RF) communication [5-7]. Recently, significant effort has been dedicated to scale down MEMS resonators into the nano regimes [8-10]; and investigate and utilize their nonlinear dynamics in applications, for example, mass sensing [8], mechanical computing [9], and NEMS electrometers $[10]$.

The dynamic behavior of M/NEMS devices can get significantly complicated and often require theoretical insight to assess design concepts before going into often complicated and time consuming fabrication and experimental characterization. Finite element models can provide satisfactory numerical results and can simulate multi-physics problems; however are often computationally expensive. In order to tackle this challenge significant focus has been dedicated to develop techniques for investigating the dynamics of MEMS structures that are efficient and can provide results with satisfactory level of accuracy [11-30]. Perturbation techniques such as the Method of Multiple Time Scales [11,12] have been effectively used to reveal the dynamic characteristics of oscillators under primary [13], secondary [14-18], parametric [19,20], and 
mixed source excitations [21,22]. Furthermore, MTS has also been used to study the selfsustained MEMS oscillators with linear feedback systems [23], nonlinearities in MEMS comb drive actuators [24], and dynamics of MEMS arch resonators [25]. MTS, although valid for weak nonlinearities and small perturbations, can reveal valuable information and insight on the dynamics of an oscillator.

Several investigations have been conducted to explore the dynamic behavior of MEMS/NEMS resonators under electrostatic excitations. These are based on the method of Multiple Time Scales (MTS) [11-25] and other techniques, such as reduced order models [26,27], and shooting techniques [28]. These studies have focused on revealing limit cycles, understanding the nonlinear behavior, investigating the stability, and studying the dynamic pullin phenomenon. Based on the findings from these investigations several applications were also proposed including realizing sharp roll-off RF filters using subharmonic resonance response [27], improving the signal-to-noise ratio using superharmonic resonance [29], and designing switches triggered by mass for sensing applications [30].

To understand the nonlinear dynamical behavior of MEMS/NEMS resonators, it is important to develop analytical models that predict the resonator's behavior under different excitation conditions. Commonly, to treat the complex parallel-plate electrostatic forcing term in the equation of motion, the forcing term is expanded in Taylor series, where some significant terms are retained and higher-order terms are dropped [19,21,24,31-34]. This is done in order to obtain a simpler equation to carry out further analysis such as bifurcation studies, stability analysis, and analytical solutions using perturbation techniques. Alternatively, the denominator of the forcing term can be cross multiplied across the equation of motion yielding a complex equation with nonlinear inertia terms [35]. Based on a reduced order model of a clamped-clamped microbeam, 
it has been demonstrated that the cross multiplication of the forcing term yields more accurate results compared to expanding the forcing term in Taylor series [35]. However, such a study has not been conducted for problems involving the Method of Multiple Time Scales, and the accuracy of both approaches and their practicality remains an open question.

In the first half of this work, we investigate the application of the two approaches, Taylor series expansion and cross multiplication of the nonlinear parallel-plate electrostatic force term, on MTS for a spring-mass-damper system under harmonic excitation in the neighborhood of primary resonance. The second half of the manuscript presents a comprehensive analytical solution based on MTS, for a simultaneous superharmonic and primary excitation of a MEMS/NEMS resonator. When a MEMS resonator is electrostatically actuated with its AC harmonic component around a certain excitation frequency, due to the quadratic form of the electrostatic voltage, the actuation is transformed into two-source excitation with one frequency around the excitation frequency and the other at twice of that $[17,18]$. In the case of excitation around half of the fundamental resonance frequency (superharmonic excitation), the contribution from the double frequency component is significant as it directly excites the resonator at its primary resonance frequency. The analytical solution obtained using MTS is then used to investigate the contribution of the superharmonic excitation component and the primary excitation component to the overall response of the resonator.

The rest paper of the paper is organized as follows. Section 2 presents the problem formulation and the mathematical model. Section 3 presents MTS analysis of the Single Degree of Freedom SDOF oscillator using the Taylor series expansion and cross multiplication techniques, and a comparison of the results. Section 4 presents the analytical solution of a MEMS resonator under the simultaneous primary and superharmonic excitation and compares 
the contribution of either excitation onto the overall response of the resonator. Section 5 presents the analytical and numerical results. Finally, Section 6 summarizes the outcomes of the study.

\section{The Model}

The non-dimensional equation of motion for the response $x(t)$ at time $t$ for an electrostatically actuated MEMS resonator using a lumped parameter model, Fig. 1, is given by [36]

$$
\begin{aligned}
\ddot{x}(t)+2 \mu \dot{x}(t)+x(t) & =\frac{\beta\left[V_{D C}+V_{A C} \cos (\Omega t)\right]^{2}}{(1-x(t))^{2}} \\
\text { where } \quad \mu & =\frac{c}{2 \sqrt{m k}} ; \beta=\frac{\grave{o} A}{2 k d^{3}}
\end{aligned}
$$

and $m$ is mass, $c$ is the damping coefficient, $k$ is the spring stiffness coefficient, $A$ is the overlap area, $d$ is the gap between electrodes, $o$ is the dielectric constant $V_{D C}$ is the DC bias, $V_{A C}$ is the AC harmonic load, and $\Omega$ is the AC frequency.

\section{Comparison of Taylor Series Expansion against Cross Multiplication}

Here, we compare applying MTS on two forms of Eq. (1): one after expanding the electrostatic force in Taylor series and the other by multiplying both sides of the equation by the denominator of the forcing. Without loss of generality, we consider a primary-resonance excitation. 


\subsection{Taylor Series Expansion}

The solution of $x$ comprises of a static component $\delta$, due to $V_{D C}$, and a dynamic component $u$; and hence can be expressed as

$$
x(t)=u(t)+\delta
$$

where

$$
\delta=\frac{\beta V_{D C}^{2}}{(1-\delta)^{2}}
$$

substituting Eq. (3) into Eq. (1), we get

$$
\ddot{u}(t)+2 \mu \dot{u}(t)+u(t)+\delta=\frac{\beta\left[V_{D C}+V_{A C} \cos (\Omega t)\right]^{2}}{(1-u(t)-\delta)^{2}}
$$

Assuming $V_{D C} \gg>V_{A C}$, expanding the right-hand side of Eq. (1) in Taylor series up to the third order in $u$, and dropping the static terms of Eq. (4), we obtain

$$
\ddot{u}(t)+2 \mu \dot{u}(t)+u(t)+\delta=\beta\left[V_{D C}^{2}+2 V_{D C} V_{A C} \cos (\Omega t)\right]\left(\frac{1}{(1-\delta)^{2}}+\frac{2 u}{(1-\delta)^{3}}+\frac{3 u^{2}}{(1-\delta)^{4}}+\frac{4 u^{3}}{(1-\delta)^{5}}+O\left(u^{4}\right)\right)
$$

Equation (6) can be written as

$$
\ddot{u}+2 \mu \dot{u}+\alpha_{q} u^{2}+\alpha_{c} u^{3}+\omega^{2} u=F \cos (\Omega t)
$$

where

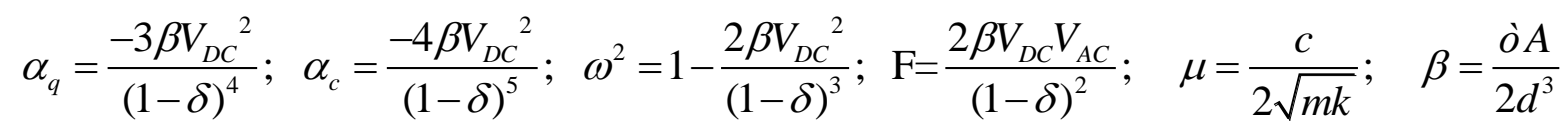

Equation (7) represents a classical single degree-of-freedom oscillator with both quadratic and cubic nonlinearities under primary resonance excitation. The frequency response equations obtained using MTS on Eq. (7) is given by $[11,12]$ 


$$
(a \mu)^{2}+\left(a \sigma-\frac{3 a^{3} \alpha_{c}}{8 \omega}+\frac{5 a^{3} \alpha_{q}^{2}}{12 \omega^{3}}\right)^{2}=\left(\frac{F}{2 \omega}\right)^{2}
$$

where $a$ is the nondimensional steady-state amplitude, and $\sigma$ is the frequency detuning parameter defines as $\sigma=\Omega-\omega$. Using the definitions from Eq. (8), we can further transform Eq. (9) into

$$
(a \mu)^{2}+\left(a \sigma-\frac{3(\delta-2) \delta a^{3}}{4(\delta-1)^{4}\left(\frac{3 \delta-1}{\delta-1}\right)^{3 / 2}}\right)^{2}=\left(\frac{\delta V a c}{\sqrt{\frac{3 \delta-1}{\delta-1}} V d c}\right)^{2}
$$

\subsection{MTS Using Force Denominator Multiplication}

Here, we multiply both sides of Eq. (5) with the denominator of the electrostatic force term, $(1-u(t)-\delta)^{2}$. Assuming $V_{D C} \gg V_{A C}$, the resulting equation is given by

$$
\left[(1-\delta)^{2}-2 u(1-\delta)+u^{2}\right](\ddot{u}+2 \mu \dot{u}+u+\delta)=\beta\left[V_{D C}^{2}+2 V_{D C} V_{A C} \cos (\Omega t)\right]
$$

dividing by $(1-\delta)^{2}$, and dropping the static term of Eq. (4), we get

$$
\begin{aligned}
\ddot{u}+2 \mu \dot{u} & +u\left(\frac{(1-3 \delta)}{(1-\delta)}-\frac{4 \mu \dot{u}}{(1-\delta)}-\frac{2 \ddot{u}}{(1-\delta)}\right)+u^{2}\left(\frac{(-2+3 \delta)}{(1-\delta)^{2}}+\frac{2 \mu \dot{u}}{(1-\delta)^{2}}+\frac{\ddot{u}}{(1-\delta)^{2}}\right) \\
+ & u^{3}\left(\frac{1}{(1-\delta)^{2}}\right)-\frac{2 V_{D C} V_{A C} \beta \cos (\Omega t)}{(1-\delta)^{2}}=0
\end{aligned}
$$

Finally, Eq. (12) can be written as

$$
\ddot{u}+2 \mu \dot{u}+u \omega^{2}+\underbrace{\alpha_{q D} u \dot{u}+\alpha_{q l} u \ddot{u}+u^{2} \alpha_{q}}_{\text {quadratic }}+\underbrace{\alpha_{c D} u^{2} \dot{u}+\alpha_{c l} u^{2} \ddot{u}+\alpha_{c} u^{3}}_{\text {cubic }}-F \cos (\Omega t)=0
$$

where 


$$
\begin{aligned}
& F=\frac{2 V_{D C} V_{A C} \beta}{(1-\delta)^{2}} ; \alpha_{c}=\frac{1}{(1-\delta)^{2}} ; \alpha_{q}=\frac{(-2+3 \delta)}{(1-\delta)^{2}} ; \alpha_{q D}=-\frac{4 \mu}{(1-\delta)} ; \alpha_{q I}=-\frac{2}{(1-\delta)} ; \alpha_{c D}=\frac{2 \mu}{(1-\delta)^{2}} ; \\
& \alpha_{c I}=\frac{1}{(1-\delta)^{2}} ; \omega^{2}=\frac{(1-3 \delta)}{(1-\delta)}
\end{aligned}
$$

We notice in Eq. (13) several inertia and damping nonlinear components.

Next, we carry out the solution of Eq. (13) using MTS. We scale Eq. (13) as follows:

$$
\ddot{u}+2 \varepsilon^{2} \mu \dot{u}+u\left(\omega^{2}+\varepsilon^{2} \alpha_{q D} \dot{u}+\alpha_{q I} \ddot{u}\right)+u^{2}\left(\alpha_{q}+\varepsilon^{2} \alpha_{c D} \dot{u}+\alpha_{c l} \ddot{u}\right)+\alpha_{c} u^{3}-\varepsilon^{3} F \cos (\Omega t)=0
$$

where $\varepsilon$ is a bookkeeping parameter. The scale chosen at Eq. (15) determines that the quadratic nonlinearity is at $\varepsilon^{2}$, the cubic nonlinearity is at $\varepsilon^{3}$, and the primary forcing and damping are at $\varepsilon^{3}$

We seek a three-term expansion of the form

$$
u(t ; \varepsilon)=\varepsilon u_{0}\left(T_{0}, T_{1}, T_{2}\right)+\varepsilon^{2} u_{1}\left(T_{0}, T_{1}, T_{2}\right)+\varepsilon^{3} u_{2}\left(T_{0}, T_{1}, T_{2}\right)
$$

where $T_{n}=\varepsilon^{n} t$.

The temporal derivatives are defined as $\frac{d}{d t}=D_{o}+\varepsilon D_{1}+\varepsilon^{2} D_{2}$, where $\frac{d}{d T_{n}}=D_{n}$. We substitute Eq. (16) into Eq. (15) to obtain

$$
\begin{aligned}
O\left(\varepsilon^{1}\right): \quad D_{o}{ }^{2} u_{0}+\omega^{2} u_{0}= & 0 \quad \Rightarrow \quad u_{0}=A\left(T_{1}, T_{2}\right) \mathrm{e}^{i \omega T_{o}}+\bar{A}\left(T_{1}, T_{2}\right) \mathrm{e}^{-i \omega T_{o}} \\
O\left(\varepsilon^{2}\right): \quad D_{o}{ }^{2} u_{1}+\omega^{2} u_{1}= & -2 D_{o} D_{1} u_{o}-\alpha_{q} u_{o}{ }^{2}-\alpha_{q I} u_{o} D_{o}{ }^{2} u_{1} \\
O\left(\varepsilon^{3}\right): \quad D_{o}{ }^{2} u_{2}+\omega^{2} u_{2}= & -\alpha_{c I} u_{o}{ }^{2} D_{o}{ }^{2} u_{o}-\alpha_{c} u_{o}{ }^{3}-2 \alpha_{q I} u_{o} D_{o} D_{1} u_{o}-\alpha_{q I} u_{o} D_{o}{ }^{2} u_{1} \\
& -\alpha_{q I} u_{0} D_{o}{ }^{2} u_{1}-2 \alpha_{q} u_{o} u_{1}-2 \mu D_{o} u_{o}-2 D_{o} D_{2} u_{o} \\
& -2 D_{o} D_{1} u_{1}-D_{1}{ }^{2} u_{o}+F \cos \left(\Omega T_{0}\right)
\end{aligned}
$$

Next, we plug Eq. (17) into Eq. (18) and obtain 


$$
\begin{aligned}
O\left(\varepsilon^{2}\right): \quad D_{o}^{2} u_{1}+\omega^{2} u_{1}= & -2 \mathrm{i} \omega D_{1} A \mathrm{e}^{\mathrm{i} T_{0} \omega}+2 \mathrm{i} \omega D_{1} \bar{A} \mathrm{e}^{-\mathrm{i} T_{0} \omega}+\left(\omega^{2} \alpha_{q I} \bar{A}^{2}-\alpha_{q} \bar{A}^{2}\right) \mathrm{e}^{-2 \mathrm{i} T_{0} \omega} \\
& +\left(\omega^{2} \alpha_{q I} A^{2}-\alpha_{q} A^{2}\right) \mathrm{e}^{2 \mathrm{i} T_{0} \omega}+2 \omega^{2} \alpha_{q I} A \bar{A}-2 \alpha_{q} A \bar{A}
\end{aligned}
$$

Eliminating the secular terms of Eq. (20) yields the following solvability condition:

$$
-2 \mathrm{i} \omega D_{1} A \mathrm{e}^{\mathrm{i} T_{0} \omega}=0 \quad \Rightarrow \quad D_{1} A=0 \quad \Rightarrow \quad A=A\left(T_{2}\right)
$$

Next, we solve Eq. (18), exclude the secular terms of Eq. (21), and obtain the particular solution $u_{1}$

$$
u_{1}=\frac{2 A\left(T_{2}\right) \bar{A}\left(T_{2}\right)\left(-\alpha_{q}+\omega^{2} \alpha_{q I}\right)}{\omega^{2}}-\frac{\bar{A}\left(T_{2}\right)^{2} \mathrm{e}^{-2 \mathrm{iT} T_{0} \omega}\left(-\alpha_{q}+\omega^{2} \alpha_{q I}\right)}{3 \omega^{2}}-\frac{A\left(T_{2}\right)^{2} \mathrm{e}^{2 \mathrm{i} T_{0} \omega}\left(-\alpha_{q}+\omega^{2} \alpha_{q I}\right)}{3 \omega^{2}}
$$

In order to express the nearness of $\Omega$ to $\omega$, we set

$$
\Omega=\omega+\varepsilon^{3} \sigma
$$

Next, we substitute Eqs. (17), (22), and (23) into Eq. (19), and obtain

$$
\begin{gathered}
3 \alpha_{c l} \omega^{2} A^{2} \bar{A}-3 \alpha_{c} A^{2} \bar{A}+\frac{1}{3} \alpha_{q I}^{2} \omega^{2} A^{2} \bar{A}+\frac{10 \alpha_{q}^{2} A^{2} \bar{A}}{3 \omega^{2}}-\frac{11}{3} \alpha_{q l} \alpha_{q} A^{2} \bar{A}-2 \mathrm{i} \omega A^{\prime} \\
-2 \mathrm{i} \mu \omega A+\frac{1}{2} F \mathrm{e}^{\mathrm{i} \sigma T_{2}}+c c=0
\end{gathered}
$$

The ()$^{\prime}$ denotes derivative with respect to $T_{2}$. We set $A\left(T_{2}\right)=\frac{1}{2} a\left(T_{2}\right) e^{i \beta\left(T_{2}\right)}, \varepsilon$ to unity, and the secular terms to zero to get the following solvability condition:

$$
-\mathrm{i} \omega a^{\prime}+a \omega \beta^{\prime}-\mathrm{i} a \mu \omega+\frac{3}{8} a^{3} \omega^{2} \alpha_{c I}-\frac{3 a^{3} \alpha_{c}}{8}+\frac{1}{24} a^{3} \omega^{2} \alpha_{q I}^{2}+\frac{5 a^{3} \alpha_{q}^{2}}{12 \omega^{2}}-\frac{11}{24} a^{3} \alpha_{q I} \alpha_{q}+\frac{1}{2} F \mathrm{e}^{\mathrm{i} \sigma T_{2}-\mathrm{i} \beta}=0
$$

Next, we set $\beta-\sigma T_{2}=\gamma$, and separate the real and imaginary parts to get the modulation equations: 


$$
\begin{array}{ll}
\operatorname{Re}: & -a \gamma^{\prime}=\frac{3}{8} a^{3} \omega \alpha_{c I}-\frac{3 a^{3} \alpha_{c}}{8 \omega}+\frac{5 a^{3} \alpha_{q}^{2}}{12 \omega^{3}}+\frac{1}{24} a^{3} \omega \alpha_{q I}^{2} \\
& -\frac{11 a^{3} \alpha_{q I} \alpha_{q}}{24 \omega}+a \sigma+\frac{F \cos (\gamma)}{2 \omega} \\
\operatorname{Im}: \quad & a^{\prime}=-a \mu-\frac{F \sin (\gamma)}{2 \omega}
\end{array}
$$

where $a$ and $\gamma$ are the nondimensional amplitude and phase, and $\sigma$ is the frequency detuning parameter. In order to obtain the steady state (equilibrium) solution, we square and add the righthand side of the modulation equations, Eq. (26) and Eq. (27), to get the frequency response equation

$$
(a \mu)^{2}+(a \sigma+\underbrace{\frac{3}{8} a^{3} \omega \alpha_{c I}-\frac{3 a^{3} \alpha_{c}}{8 \omega}}_{\text {cubic }}+\underbrace{\frac{1}{24} a^{3} \omega \alpha_{q I}^{2}-\frac{11 a^{3} \alpha_{q I} \alpha_{q}}{24 \omega}+\frac{5 a^{3} \alpha_{q}^{2}}{12 \omega^{3}}}_{\text {quadratic }})^{2}=\left(\frac{F}{2 \omega}\right)^{2}
$$

Next, we substitute Eq. (4) and the coefficients from Eq. (14) into Eq. (29) to get

$$
(a \mu)^{2}+\left(a \sigma-\frac{3(\delta-2) \delta a^{3}}{4(\delta-1)^{4}\left(\frac{3 \delta-1}{\delta-1}\right)^{3 / 2}}\right)^{2}=\left(\frac{\delta V a c}{\sqrt{\frac{3 \delta-1}{\delta-1} V d c}}\right)^{2}
$$

We notice that Eq. (10) and Eq. (29) are identical equations. This shows that whether the Taylor series expansion is used or the force denominator multiplication technique is used, the resulting equations are the same. This holds as long as the order of retained terms is the same in both techniques. However, one can note that it is easier to use the Taylor series expansion as it yields a simpler equation of motion, which is easier to analyze in MTS. 


\section{Simultaneous Superharmonic and Primary Resonance Excitation}

Next, we apply the method of multiple scales to analyze the dynamic response of a MEMS resonator under the simultaneous superharmonic and primary resonance excitation.

The parallel plate electrostatic harmonic forcing term is quadratic in nature, Eq. (1). Because of this, the beam resonator experiences a simultaneous excitation at the frequency of excitation $\Omega$ due to the $2 V_{D C} V_{A C} \cos (\Omega t)$ component and at twice of that due to the $\frac{V_{A C}{ }^{2}}{2} \cos (2 \Omega t)$ component. Commonly, it is assumed that $V_{D C} \gg V_{A C}$ and the $\frac{V_{A C}{ }^{2}}{2} \cos (2 \Omega t)$ component is neglected. However, there are many applications where $V_{A C}$ and $V_{D C}$ are of the same order. Hence, it is important to take this excitation component at twice the resonance frequency into account to predict the response of the resonator accurately $[17,18]$. Furthermore, in the case of a superharmonic excitation, where the excitation frequency is deliberately at half the resonance frequency $\Omega \approx \omega / 2$, for example in [27,28], the $\cos (2 \Omega t)$ component excites the resonator at the primary resonance frequency, which cannot be negligible and must be taken into account.

In this section an analytical solution using MTS is presented that takes into account both excitation components. The derived solution is valid for a generic electrostatic loading case. The results will be further analyzed to see the contribution of each of the excitation component in the resonator's overall response.

In order to explore the MTS solution for a simultaneous superharmonic and primary excitation, we use the Taylor series expansion method. Expanding the right-hand side of Eq. (5) in Taylor series, we get 


$$
\begin{aligned}
\ddot{u}(t)+2 \mu \dot{u}(t)+u(t)+\delta= & \beta\left(V_{D C}^{2}+\frac{V_{A C}{ }^{2}}{2}+2 V_{D C} V_{A C} \cos (\Omega t)+\frac{V_{A C}^{2}}{2} \cos (2 \Omega t)\right) \\
& \times\left(\frac{1}{(1-\delta)^{2}}+\frac{2 u}{(1-\delta)^{3}}+\frac{3 u^{2}}{(1-\delta)^{4}}+\frac{4 u^{3}}{(1-\delta)^{5}}+O\left(u^{4}\right)\right)
\end{aligned}
$$

It is important to note here that the static deflection in this case is obtained by solving $\delta=\frac{\beta\left(V_{D C}^{2}+\frac{V_{A C}^{2}}{2}\right)}{(1-\delta)^{2}}$. This shows the contribution from the AC forcing term to the static solution. The equation of motion is given by

$$
\begin{aligned}
& \qquad \ddot{u}+2 \mu \dot{u}+\alpha_{q} u^{2}+\alpha_{c} u^{3}+\omega^{2} u=F_{p} \cos (\Omega t)+F_{s} \cos (2 \Omega t)+F_{p p a r 1} \cos (\Omega t) u+F_{p p a r 2} \cos (\Omega t) u^{2} \\
& \text { where } \quad \alpha_{q}=\frac{-3 \beta V_{e f f}}{(1-\delta)^{4}} ; \quad \alpha_{c}=\frac{-4 \beta V_{e f f}}{(1-\delta)^{5}} ; \omega^{2}=1-\frac{2 \beta V_{e f f}}{(1-\delta)^{3}} ; F_{p}=\frac{2 \beta V_{D C} V_{A C}}{(1-\delta)^{2}} ; F_{s}=\frac{\beta V_{A C}^{2}}{2(1-\delta)^{2}} ; \\
& F_{p p a r 1}=\frac{4 \beta V_{A C}^{2}}{(1-\delta)^{3}} ; F_{p p a r 2}=\frac{6 \beta V_{A C}^{2}}{(1-\delta)^{4}} ; \quad V_{e f f}=V_{D C}^{2}+\frac{V_{A C}^{2}}{2}
\end{aligned}
$$

The higher order parametric terms are retained here in order to improve accuracy of the solution $[17,18]$.

We scale Eq. (31) as follows:

$$
\begin{aligned}
\ddot{u}+2 \varepsilon^{2} \mu \dot{u}+\alpha_{q} u^{2}+\alpha_{c} u^{3}+\omega^{2} u= & \varepsilon F_{p} \cos (\Omega t)+\varepsilon^{3} F_{s} \cos (2 \Omega t)+\varepsilon F_{p p a r 1} \cos (\Omega t) u \\
& +\varepsilon F_{p p a r 2} \cos (\Omega t) u^{2}
\end{aligned}
$$

where $\varepsilon$ is a bookkeeping parameter. The scale chosen at Eq. (33) determines that the quadratic nonlinearity is at $\varepsilon^{2}$, the cubic nonlinearity is at $\varepsilon^{3}$, the secondary forcing component and damping are at $\varepsilon^{3}$, and the primary forcing component is at $\varepsilon$.

We seek a three-term expansion of the form 


$$
u(t ; \varepsilon)=\varepsilon u_{0}\left(T_{0}, T_{1}, T_{2}\right)+\varepsilon^{2} u_{1}\left(T_{0}, T_{1}, T_{2}\right)+\varepsilon^{3} u_{2}\left(T_{0}, T_{1}, T_{2}\right)
$$

where $T_{n}=\varepsilon^{n} t$.

The temporal derivatives are defined as $\frac{d}{d t}=D_{o}+\varepsilon D_{1}+\varepsilon^{2} D_{2}$, where $\frac{d}{d T_{n}}=D_{n}$. We substitute Eq. (34) into Eq. (33) to obtain

$$
O\left(\varepsilon^{1}\right): \quad D_{o}^{2} u_{0}+\omega^{2} u_{0}=F_{p} \cos (2 \Omega t) \quad \Rightarrow \quad u_{0}=A \mathrm{e}^{i \omega T_{o}}+\lambda_{p} \mathrm{e}^{i \Omega T_{o}}+c c
$$

where $\lambda_{p}=\frac{F_{p}}{\omega^{2}-\Omega^{2}}$ and $A \Rightarrow A\left(T_{1}, T_{2}\right)$

$$
\begin{aligned}
O\left(\varepsilon^{2}\right): \quad D_{o}^{2} u_{1}+\omega^{2} u_{1}= & -2 D_{o} D_{1} u_{o}-\alpha_{q} u_{o}^{2}+F_{p p a r 1} \cos \left(\Omega T_{o}\right) u_{o} \\
O\left(\varepsilon^{3}\right): \quad D_{o}^{2} u_{2}+\omega^{2} u_{2}= & -2 D_{o} D_{1} u_{1}-2 D_{o} D_{2} u_{o}-2 \mu D_{o} u_{o}-D_{1}^{2} u_{o}-2 \alpha_{q} u_{o} u_{1}-\alpha_{c} u_{o}^{3} \\
& +F_{p} \cos \left(\Omega T_{0}\right)+F_{p p a r 1} \cos \left(\Omega T_{0}\right) u_{1}+F_{p p a r 2} \cos \left(2 \Omega T_{0}\right) u_{o}^{2}
\end{aligned}
$$

In order to express the nearness of $\Omega$ to $\omega$, we set

$$
\Omega=\frac{1}{2} \omega+\varepsilon^{2} \sigma
$$

Next, we plug Eqs. (35) and (38) into Eq. (36), obtain the secular terms, and set them equal to zero yielding the following solvability condition:

$$
-\lambda_{p}^{2} \alpha_{q} \mathrm{e}^{2 \mathrm{i} \sigma T_{2}}-2 \mathrm{i} \omega D_{1} A+\frac{1}{2} F_{p p a r 1} \lambda_{p} \mathrm{e}^{2 \mathrm{i} \sigma T_{2}}+c c=0
$$

From Eq. (39) we get

$$
\begin{aligned}
& D_{1} A=\frac{\mathrm{i} \lambda_{p}^{2} \alpha_{q} \mathrm{e}^{2 \mathrm{i} \sigma T_{2}}}{2 \omega}-\frac{\mathrm{i} F_{p p a r 1} \lambda_{p} \mathrm{e}^{2 \mathrm{i} \sigma T_{2}}}{4 \omega} \\
& \mathrm{D}_{1} \bar{A}=\frac{\mathrm{i} F_{p p a r 1} \lambda_{p} \mathrm{e}^{-2 \mathrm{i} \sigma T_{2}}}{4 \omega}-\frac{\mathrm{i} \lambda_{p}^{2} \alpha_{q} \mathrm{e}^{-2 \mathrm{i} \sigma T_{2}}}{2 \omega} \\
& \mathrm{D}_{1}^{2} A=0
\end{aligned}
$$


Next, we solve Eq. (36) excluding the secular terms of Eq. (39) to get the particular solution $u_{1}$

$$
\begin{aligned}
& u_{1}=\frac{\left(F_{p p a r 1}-4 \lambda_{p} \alpha_{q}\right) \bar{A} \mathrm{e}^{-\mathrm{i}(\omega-\Omega) T_{0}}}{2 \Omega(2 \omega-\Omega)}+\frac{\left(4 \lambda_{p} \alpha_{q}-F_{p p a r 1}\right) \bar{A} \mathrm{e}^{-\mathrm{i}(\omega+\Omega) T_{0}}}{2 \Omega(2 \omega+\Omega)}-\frac{2 \alpha_{q} A \bar{A}}{\omega^{2}}+\frac{\alpha_{q} \bar{A}^{2} \mathrm{e}^{-2 \mathrm{i} \omega T_{0}}}{3 \omega^{2}} \\
& +\frac{\left(F_{p p a r 1}-4 \lambda_{p} \alpha_{q}\right) A \mathrm{e}^{\mathrm{i}(\omega-\Omega) T_{0}}}{2 \Omega(2 \omega-\Omega)}+\frac{\left(4 \lambda_{p} \alpha_{q}-F_{p p a r 1}\right) A \mathrm{e}^{\mathrm{i}(\omega+\Omega) T_{0}}}{2 \Omega(2 \omega+\Omega)}+\frac{\alpha_{q} A^{2} \mathrm{e}^{2 \mathrm{i} \omega T_{0}}}{3 \omega^{2}}+\frac{F_{p p a r 1} \lambda_{p}}{\omega^{2}}-\frac{2 \lambda_{p}^{2} \alpha_{q}}{\omega^{2}}
\end{aligned}
$$

Then, we substitute Eqs. (35), and (41) into Eq. (37), obtain the secular terms, and set them equal to zero

$$
\begin{aligned}
& \left(\begin{array}{l}
-6 \lambda_{p}^{2} \alpha_{c}-\frac{2 \lambda_{p} F_{p p a r 1} \alpha_{q}}{\omega^{2}}-\frac{2 \lambda_{p} F_{p p a r 1} \alpha_{q}}{\Omega(2 \omega-\Omega)}+\frac{2 \lambda_{p} F_{p p a r 1} \alpha_{q}}{\Omega(2 \omega+\Omega)}+2 \lambda_{p} F_{p p a r 2}+\frac{F_{p p a r 1}^{2}}{4 \Omega(2 \omega-\Omega)} \\
-\frac{F_{p p a r 1}^{2}}{4 \Omega(2 \omega+\Omega)}-2 \mathrm{i} \mu \omega+\frac{4 \lambda_{p}^{2} \alpha_{q}^{2}}{\omega^{2}}+\frac{4 \lambda_{p}^{2} \alpha_{q}^{2}}{\Omega(2 \omega-\Omega)}-\frac{4 \lambda_{p}^{2} \alpha_{q}^{2}}{\Omega(2 \omega+\Omega)}
\end{array}\right) A \\
& +\left(\frac{10 \alpha_{q}^{2}}{3 \omega^{2}}-3 \alpha_{c}\right) A^{2} \bar{A}-2 \mathrm{i} \omega D_{2} A-D_{1}^{2} A+\frac{1}{2} F_{p} \mathrm{e}^{\mathrm{i} \sigma T_{2}}+c c=0
\end{aligned}
$$

From Eq. (42) we get

$$
\begin{aligned}
D_{2} A= & \left(\begin{array}{l}
\frac{3 \mathrm{i} \alpha_{c} \lambda_{p}^{2}}{\omega}+\frac{\mathrm{i} F_{p p a r 1} \lambda_{p} \alpha_{q}}{\omega^{3}}+\frac{\mathrm{i} F_{p p a r 1} \lambda_{p} \alpha_{q}}{\omega \Omega(2 \omega-\Omega)}-\frac{\mathrm{i} F_{p p a r 1} \lambda_{p} \alpha_{q}}{\omega \Omega(2 \omega+\Omega)}-\frac{\mathrm{i} F_{p p a r} \lambda_{p}}{\omega} \\
-\frac{\mathrm{i} F_{p p a r 1}^{2}}{8 \omega \Omega(2 \omega-\Omega)}+\frac{\mathrm{i} F_{p p a r 1}^{2}}{8 \omega \Omega(2 \omega+\Omega)}-\mu-\frac{2 \mathrm{i} \lambda_{p}^{2} \alpha_{q}^{2}}{\omega^{3}}-\frac{2 \mathrm{i} \lambda_{p}^{2} \alpha_{q}^{2}}{\omega \Omega(2 \omega-\Omega)}+\frac{2 \mathrm{i} \lambda_{p}^{2} \alpha_{q}^{2}}{\omega \Omega(2 \omega+\Omega)}
\end{array}\right) A \\
& +\left(\frac{3 \mathrm{i} \alpha_{c}}{2 \omega}-\frac{5 \mathrm{i} \alpha_{q}^{2}}{3 \omega^{3}}\right) A^{2} \bar{A}+\frac{\mathrm{i} D_{1}^{2} A}{2 \omega}-\frac{\mathrm{i} F_{s} \mathrm{e}^{2 \mathrm{i} \sigma T_{2}}}{4 \omega}
\end{aligned}
$$

Next, we use the method of reconstitution

$$
\frac{d A}{d t}=D_{1} A+D_{2} A
$$

and setting $\varepsilon$ to unity

$$
A\left(T_{1}, T_{2}\right)=\frac{1}{2} a(t) e^{i \beta(t)}
$$


Using the definitions from Eqs. (40), (43), (44), and (45) into Eq. (42) we obtain

$$
\begin{aligned}
\frac{a^{\prime}}{2}+\frac{1}{2} \mathrm{i} a \beta= & -\frac{a \mu}{2}-\frac{\mathrm{i} a F_{p p a r 1}^{2}}{16 \omega(2 \omega-\Omega) \Omega}+\frac{\mathrm{i} a F_{p p a r}^{2}}{16 \omega \Omega(2 \omega+\Omega)}-\frac{\mathrm{ie}^{-\mathrm{i} \beta+2 \mathrm{i} \sigma T_{2}} F_{s}}{4 \omega} \\
& +\frac{3 \mathrm{i} a^{3} \alpha_{c}}{16 \omega}-\frac{5 \mathrm{i} a^{3} \alpha_{q}^{2}}{24 \omega^{3}}-\frac{\mathrm{ie}^{-\mathrm{i} \beta+2 \mathrm{i} \sigma T_{2}} F_{p p a r 1} \lambda_{p}}{4 \omega}-\frac{\mathrm{i} a F_{p p a r} \lambda_{p}}{2 \omega}-\frac{\mathrm{i} a \alpha_{q}^{2} \lambda_{p}^{2}}{\omega^{3}} \\
& +\frac{\mathrm{i} a F_{p p a r 1} \alpha_{q} \lambda_{p}}{2 \omega(2 \omega-\Omega) \Omega}-\frac{\mathrm{i} a F_{p p a r 1} \alpha_{q} \lambda_{p}}{2 \omega \Omega(2 \omega+\Omega)}+\frac{3 \mathrm{i} a \alpha_{c} \lambda_{p}^{2}}{2 \omega}+\frac{\mathrm{ie}{ }^{-\mathrm{i} \beta+2 \mathrm{i} \sigma T_{2}} \alpha_{q} \lambda_{p}^{2}}{2 \omega} \\
& -\frac{\mathrm{i} a \alpha_{q}^{2} \lambda_{p}^{2}}{\omega(2 \omega-\Omega) \Omega}+\frac{\mathrm{i} a \alpha_{q}^{2} \lambda_{p}^{2}}{\omega \Omega(2 \omega+\Omega)}+\frac{\mathrm{i} a F_{p p a r 1} \alpha_{q} \lambda_{p}}{2 \omega^{3}}
\end{aligned}
$$

where ()$^{\prime}$ is the derivative with respect to $t$. Next, we set $\beta-\sigma T_{2}=\gamma$, and separate the real and imaginary parts to get the modulation equations

$$
\operatorname{Re}: \quad \frac{a^{\prime}}{2}=-\frac{a \mu}{2}-\frac{\lambda_{p} F_{p p a r 1} \sin (\gamma)}{4 \omega}-\frac{F_{s} \sin (\gamma)}{4 \omega}+\frac{\lambda_{p}^{2} \alpha_{q} \sin (\gamma)}{2 \omega}
$$

$$
\operatorname{Im}: \quad \frac{a \gamma^{\prime}}{2}=a \sigma-\frac{3 a^{3} \alpha_{c}}{16 \omega}+\frac{5 a^{3} \alpha_{q}^{2}}{24 \omega^{3}}-\frac{3 a \alpha_{c} \lambda_{p}^{2}}{2 \omega}-\frac{a F_{p p a r 1} \lambda_{p} \alpha_{q}}{2 \omega^{3}}-\frac{a F_{p p a r 1} \lambda_{p} \alpha_{q}}{2 \omega \Omega(2 \omega-\Omega)}
$$

$$
\begin{aligned}
& +\frac{a F_{p p a r 1} \lambda_{p} \alpha_{q}}{2 \omega \Omega(2 \omega+\Omega)}+\frac{a F_{p p a r} \lambda_{p}}{2 \omega}+\frac{a F_{p p a r 1}^{2}}{16 \omega \Omega(2 \omega-\Omega)}-\frac{a F_{p p a r 1}^{2}}{16 \omega \Omega(2 \omega+\Omega)} \\
& +\frac{a \lambda_{p}^{2} \alpha_{q}^{2}}{\omega^{3}}+\frac{a \lambda_{p}^{2} \alpha_{q}^{2}}{\omega \Omega(2 \omega-\Omega)}-\frac{a \lambda_{p}^{2} \alpha_{q}^{2}}{\omega \Omega(2 \omega+\Omega)}+\frac{F_{p p a r 1} \lambda_{p} \cos (\gamma)}{4 \omega}
\end{aligned}
$$

$$
+\frac{F_{s} \cos (\gamma)}{4 \omega}-\frac{\lambda_{p}^{2} \alpha_{q} \cos (\gamma)}{2 \omega}
$$

The steady state (equilibrium) solution is then obtained by solving algebraically the righthand side of the modulation equations. Here $a$ and $\gamma$ are the nondimensional amplitude and phase, $\sigma$ is the frequency detuning parameter, and $\Omega$ is given by the definition in Eq. (38) for $\varepsilon=1$. 


\section{Results}

In this section, the frequency responses of a microstructure for simultaneous excitation of superharmonic and primary excitation using Eq. (47) and Eq. (48) are presented and compared with the numerical results obtained using the long time integration (LTI) of Eq. (1). As a case study, we assume a microstructure such that $\beta=7.14 \times 10^{-5} \mathrm{~V}^{-2}$, while the rest of parameters in Eq. (32) are computed based on the assumed voltage loads. It is important to note that the excitation frequency here is close to half of the resonator's primary resonance frequency $\left(\omega_{n}\right)$, i.e., $\Omega \approx \frac{1}{2} \omega_{n}$. Hence, the frequency component $\cos (2 \Omega t)$ excites the resonator around its natural frequency (primary excitation component), while the frequency component $\cos (\Omega t)$ excites the resonator around half of its natural frequency (superharmonic excitation component).

Figure 2 shows the results for various combinations of DC and AC voltage loads. Good agreement with the LTI solution of Eq. (1) is observed for various cases of loading conditions. It is observed that the analytical solution (using MTS) deviates more from the numerical solution (using LTI) for the cases when the superharmonic excitation term $(\cos (\Omega t))$ is stronger than the primary excitation term $(\cos (2 \Omega t))$, Fig. 2(a)-(b). However, for the case where the primary excitation term is stronger than the superharmonic excitation term, the solutions match better, Fig. 2(c). This indicates that a direct excitation term associated with $(\cos (2 \Omega t))$ term is enough to capture the contribution from the primary excitation component, however, more parametric terms associated with the excitation component $(\cos (\Omega t))$ are required to completely capture the contribution of the superharmonic excitation. However, obtaining such a solution can add unnecessary complexity as this level of accuracy is acceptable and gives good qualitative agreement. It is also important to mention here that a certain deviation from the numerical 
solution will always be there due to the fact that a truncated from of Eq. (1) given by Eq. (31), where higher order terms are dropped, is used for MTS. In order to verify this, numerical solution of Eq. (31) was carried out using LTI and compared with the solutions obtained through MTS and LTI of Eq. (1), Fig. 2(c). An excellent match is noticed between the MTS solution and the numerical solution of the truncated Eq. (31).

Next, the analytical solution is used to assess the contributions from each of the primary $\cos (2 \Omega t)$ and superharmonic $\cos (\Omega t)$ excitation components to the overall resonator's response. A response to only superharmonic excitation is obtained by setting $F_{s}=0$ in Eq. (47) and Eq. (48), while response to only primary excitation is obtained by setting $F_{p}=\lambda_{p}=F_{p p a r 1}=F_{p p a r 2}=0$. Figure 3 shows this comparison for the frequency response curves of Fig. 2.

The frequency response curves of Fig. 3 show that the contribution from the primary excitation component $(\cos (2 \Omega t))$ is always higher than the superharmonic excitation component $(\cos (\Omega t))$. This is expected as the excitation around the primary resonance frequency is much stronger than the excitation around the secondary resonances. This indicates that the reported superharmonic excitation at $\Omega \approx \frac{1}{2} \omega_{n}$ in $[14,27,28]$ was strongly influenced by the $\cos (2 \Omega t)$ term, leading effectively to a primary-resonance dominated response. When the superharmonic excitation term at $\cos (\Omega t)$ is of comparable strength to the primary excitation term at $\cos (2 \Omega t)$, the contribution from both components must be taken into account to get the actual response of the resonator, Fig 3(a)-(b). However, in scenarios when the primary excitation term is much stronger than the superharmonic excitation term, the former can be neglected and a response of the resonator to only primary excitation term provides accurate results, Fig 3(c). 


\section{Conclusions}

Modeling the dynamic behavior of an electrostatically actuated resonator using the perturbation method of Multiple Time Scales is investigated. MTS solution is carried out for a SDOF spring-mass-damper system, electrostatically excited at primary resonance, using two approaches aimed at simplifying the nonlinear parallel-plate electrostatic force term, 1) expanding the force term in Taylor series expansion, 2) cross-multiplying the equation of motion with the denominator of the electrostatic force term. It is observed that with a consistent truncation of the higher-order terms the final frequency response equations obtained through MTS are the same for both techniques. It is concluded that the choice of treating these complex nonlinearities either using Taylor series expansion or multiplication of the denominator of the force term is unimportant. However, MTS analysis via the multiplication method is more complex compared to the Taylor series expansion method. Hence, Taylor series expansion of the parallel-plate electrostatic forcing term is recommended. Next, using the Taylor series expansion and keeping the direct excitation as well as some of the parametric excitation terms, an MTS solution is carried out to predict the response of a resonator under simultaneous superharmonic and primary excitation. A strong agreement between the frequency response results obtained through MTS and numerical results obtain using LTI of the equation of motion is reported. It is observed that for a MEMS resonator excited near half of its fundamental resonance frequency (superharmonic excitation), the primary excitation component arising due to the quadratic nature of the electrostatic force contributes more to the overall response of the resonator. Hence, the overall response of the resonator for a superharmonic excitation resembles closely the primary resonance response. 


\section{Acknowledgement}

This publication is based upon work supported by the King Abdullah University of Science and Technology (KAUST) research funds.

\section{Conflict of Interest}

The authors declare that they have no conflict of interest.

\section{References}

[1] Rhoads, J. F., Shaw, S. W., and Turner, K. L., 2010, "Nonlinear dynamics and its applications in micro-and nanoresonators,” J. Dynam. Syst. Meas. Cont., 132, pp. 034001.

[2] Zhang, W., and Turner, K. L., 2005, “Application of parametric resonance amplification in a singlecrystal silicon micro-oscillator based mass sensor," Sens. Actuat. A: Phys., 122, pp. 23-30.

[3] Hafiz, M. A. A., Kosuru, L., and Younis, M. I., 2016, "Microelectromechanical reprogrammable logic device," Nat. comm., 7, pp. 11137.

[4] Ilyas, S., Jaber, N., and Younis, M. I., 2017, "MEMS logic using mixed-frequency excitation,” J. Microelectromech. Syst., 26, pp. 1140-1146.

[5] Wong, A. C., and Nguyen, C. C., 2004, "Micromechanical mixer-filters ("mixlers")," J. Microelectromech. Syst., 13, pp. 100-112.

[6] Ilyas, S., Chappanda, K. N., and Younis, M. I., "Exploiting nonlinearities of micro-machined resonators for filtering applications,” Appl. Phys. Lett., 110, pp. 253508.

[7] Pourkamali, S., and Ayazi, F., 2005, "Electrically coupled MEMS bandpass filters: Part I: With coupling element," Sens. Actuat. A: Phys., 122, pp. 307-316. 
[8] Kacem, N., Hentz, S., Pinto, D., Reig, B., and Nguyen, V., 2009, "Nonlinear dynamics of nanomechanical beam resonators: improving the performance of NEMS-based sensors," Nanotech., 20, pp. 275501.

[9] Guerra, D. N., Bulsara, A. R., Ditto, W. L., Sinha, S., Murali, K., and Mohanty, P., 2010, “A noiseassisted reprogrammable nanomechanical logic gate,” Nan. Lett., 10, pp. 1168-1171.

[10]Jalil, J., Zhu, Y., Ekanayake, C., and Ruan, Y., 2017, Sensing of single electrons using micro and nano technologies: a review," Nanotech., 28, pp. 142002.

[11]Nayfeh, A. H., 2011, Introduction to perturbation techniques, John Wiley and Sons.

[12] Nayfeh, A. H., and Mook, D. T., 2008, Nonlinear oscillations. John Wiley and Sons.

[13] Younis, M. I., and Nayfeh, A. H. A study of the nonlinear response of a resonant microbeam to an electric actuation. Nonlin. Dynam., 31, pp. 91-117.

[14] Abdel-Rahman, E. M., Nayfeh, A. H., 2003, "Secondary resonances of electrically actuated resonant microsensors," J. Micromech. Microeng., 13, pp. 491.

[15]Nayfeh, A. H., "Quenching of primary resonance by a superharmonic resonance," 1984, J. Sound Vib., 92, pp. 363-377.

[16] Nayfeh, A. H., 1983, "The response of single degree-of-freedom systems with quadratic and cubic non-linearities to a subharmonic excitation,” J. Sound Vib., 89, pp. 457-470.

[17]Ilyas, S., Alfosail, F., and Younis, M. I., "On the Response of MEMS Resonators under Generic Electrostatic Loadings: Theoretical Analysis,” Nonlinear Dynam., Submitted (2018).

[18]Ilyas, S., Alfosail, F., and Younis, M. I., "On the Response of MEMS Resonators under Generic Electrostatic Loadings: Experiments and Applications,” Nonlinear Dynam., Submitted (2018).

[19]Zhang, W. M., and Guang M., 2007, "Nonlinear dynamic analysis of electrostatically actuated resonant MEMS sensors under parametric excitation,” IEEE sensors., 7, pp. 370-380.

[20]Caruntu, D. I., and Martinez, I., 2014, "Reduced order model of parametric resonance of electrostatically actuated MEMS cantilever resonators," Int. J. Non-Linear Mech., 66, pp. 28-32. 
[21]Elnaggar, A. E., El-Bassiouny, and A., Mosa, G., 2014, "Perturbation analysis of an electrostatic Micro-Electro-Mechanical System (MEMS) subjected to external and non-linear parametric excitations,' Int. J. Basic Appl. Sci., 3, pp. 209-223.

[22]Nayfeh, A. H., 1985, "The response of non-linear single-degree-of-freedom systems to multifrequency excitations,” J. Sound Vib., 102, pp. 403-414.

[23]Chen, C., Zanette, D. H., Guest, J. R., Czaplewski, D. A., and López, D., 2016, "Self-sustained micromechanical oscillator with linear feedback,” Physical Rev. Lett., 117, pp. 017203.

[24]Elshurafa, A. M., Khirallah, K., Tawfik, H. H., Emira, A., Aziz, A. K. A., and Sedky, S. M., 2011, "Nonlinear dynamics of spring softening and hardening in folded-MEMS comb drive resonators," J. Microelectromech. Syst., 20, pp. 943-958.

[25] Ouakad, H. M., and Younis, M. I., 2010, "The dynamic behavior of MEMS arch resonators actuated electrically.” Int. J. Non-Linear Mech., 45, pp. 704-713.

[26] Ouakad, H. M., and Younis, M. I., 2010, "Nonlinear dynamics of electrically actuated carbon nanotube resonators,” J. Comput. Nonlin. Dyn., 5, pp. 011009.

[27]Nayfeh, A. H., and Younis, M. I., 2005, "Dynamics of MEMS resonators under superharmonic and subharmonic excitations,” J. Micromech. Microeng., 15, pp. 1840.

[28] Alsaleem, F. M., Younis, M. I., and Ouakad, H. M., 2009, “On the nonlinear resonances and dynamic pull-in of electrostatically actuated resonators,” J. Micromech. Microeng., 19, pp. 045013.

[29]Jin, Z., and Wang, Y., 1998, “Electrostatic resonator with second superharmonic resonance,” Sens. Actuat. A: Phys., 64, pp. 273-279.

[30] Younis, M. I., and Alsaleem, F., 2009, "Exploration of new concepts for mass detection in electrostatically-actuated structures based on nonlinear phenomena,” J. Comput. Nonlin. Dyn., 4, pp. 021010.

[31] Ouakad, H. M., Alofi, A. M., and Nayfeh, A. H., 2017, "Dynamic Analysis of Multilayers Based MEMS Resonators," Mathematical Problems in Engineering. 
[32] Azizi, S., Chorsi, M. T., and Nejad, F. B., 2016, "On the secondary resonance of a MEMS resonator: A conceptual study based on shooting and perturbation methods," Int. J. Non-Linear Mech., 82, pp.59-68.

[33]Rhoads, J. F., Shaw, S. W., and Turner, K. L., 2006, “The nonlinear response of resonant microbeam systems with purely-parametric electrostatic actuation,” J. Micromech. Microeng., 16, pp. 890.

[34]Elnaggar, A. M., Khalil, K. M., and Rahby, A. S., 2016, "Harmonic solution of a weakly nonlinear second order differential equation governed the motion of a TM-AFM cantilever," British Journal of Mathematics and Computer Science, 15, pp. 1-11.

[35] Younis, M. I., Abdel-Rahman, E. M., and Nayfeh, A. H., 2003, "A reduced-order model for electrically actuated microbeam-based MEMS,” J.Microelectromech. Syst., 12, pp. 672-680.

[36] Younis, M. I., 2011, MEMS linear and nonlinear statics and dynamics. Springer Science and Business Media. 


\section{Figures}

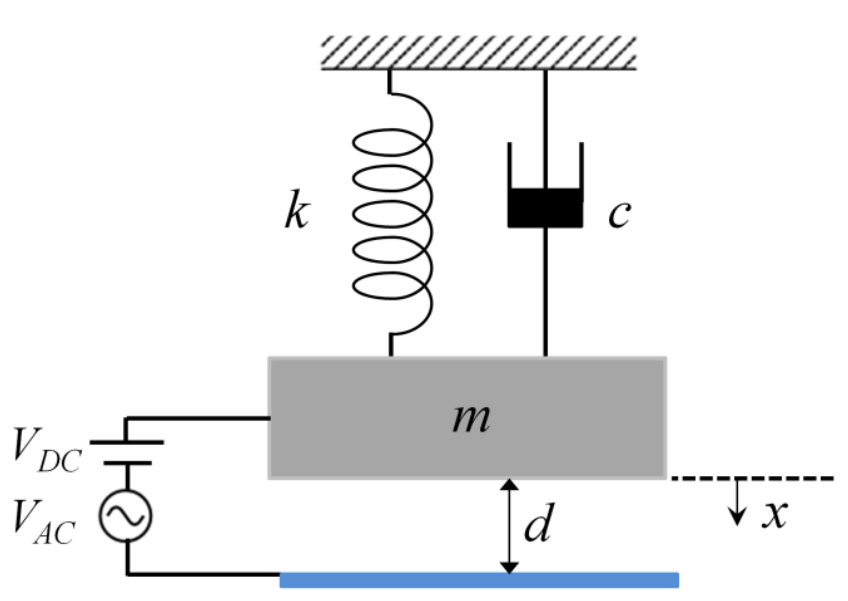

Fig. 1 Schematic of a single degree-of-freedom (SDOF) spring-mass-damper system representing a MEMS microbeam resonator under parallel-plate electrostatic actuation.

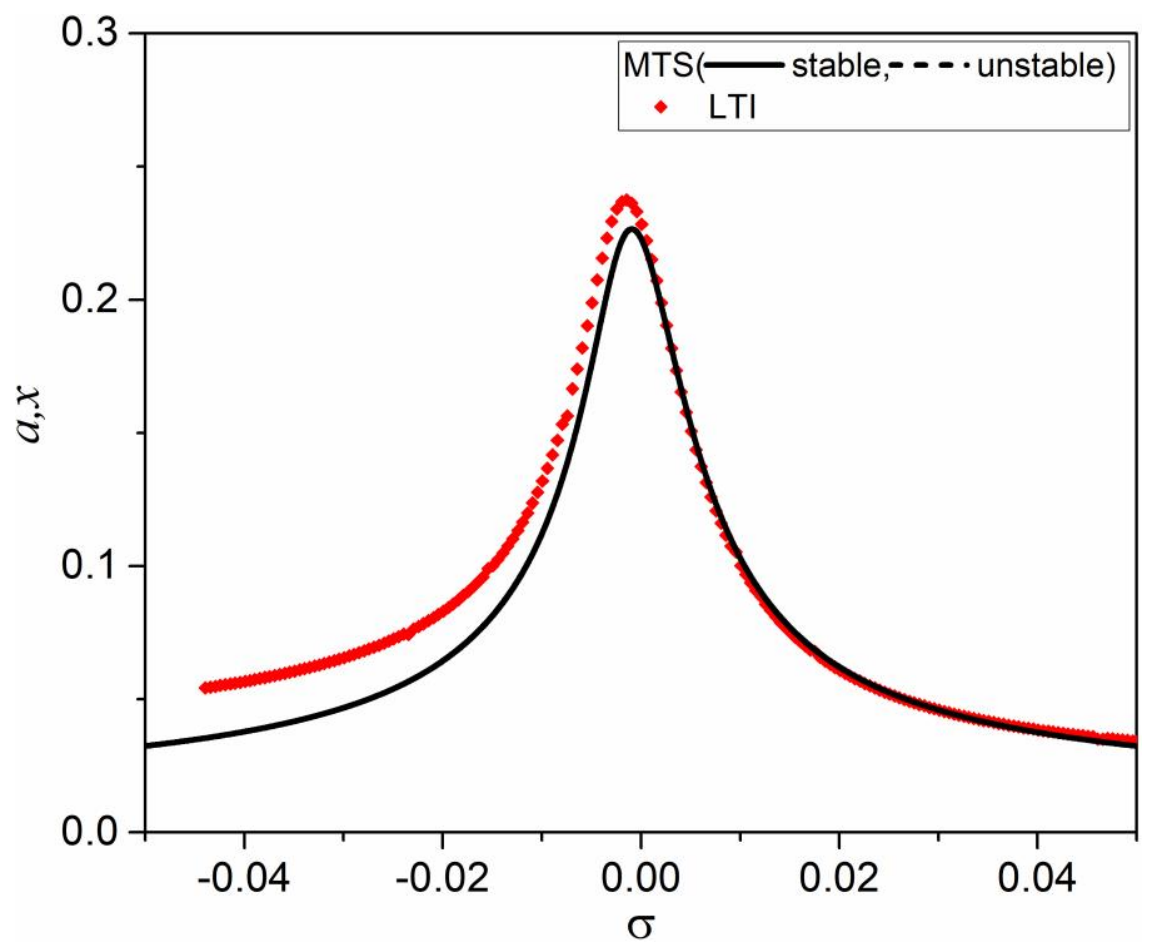

(a) 


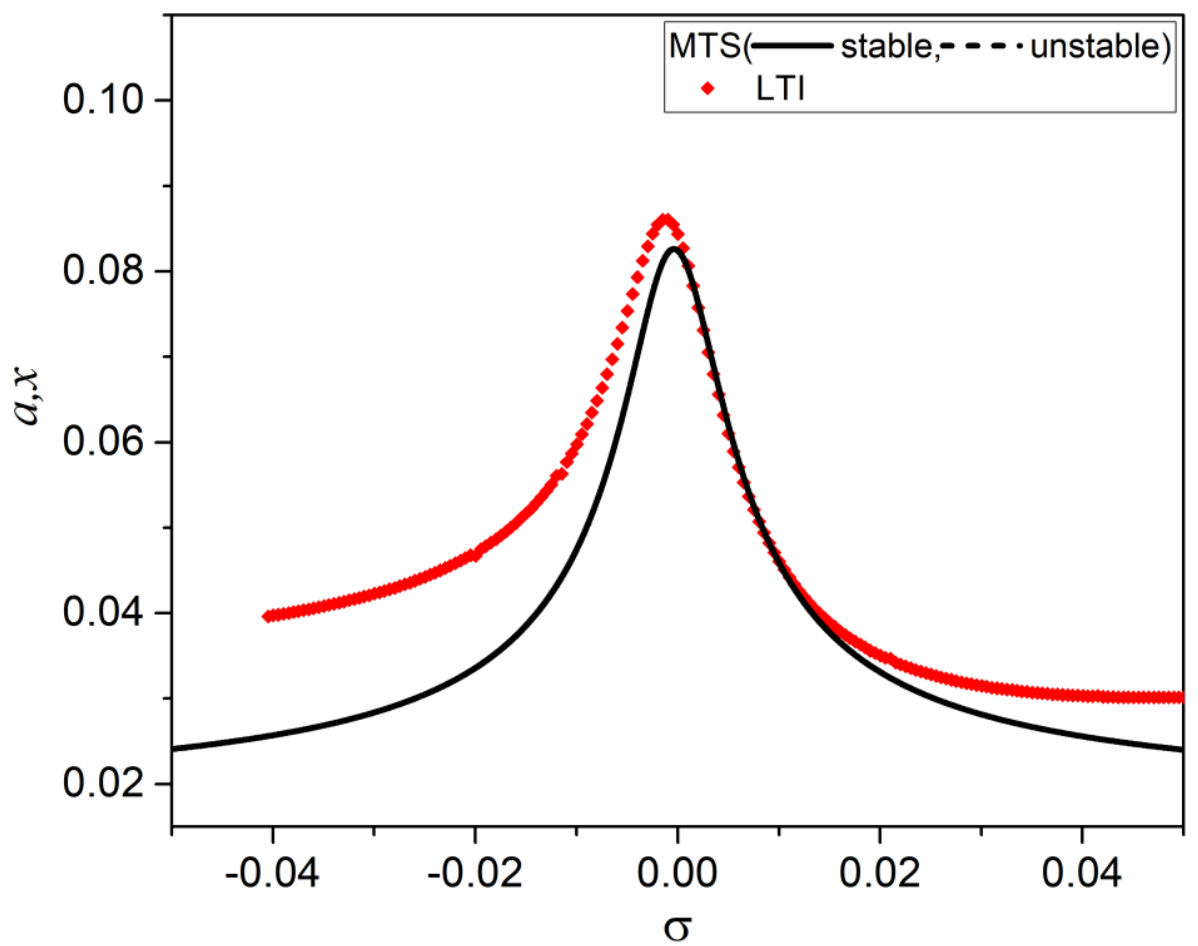

(b)

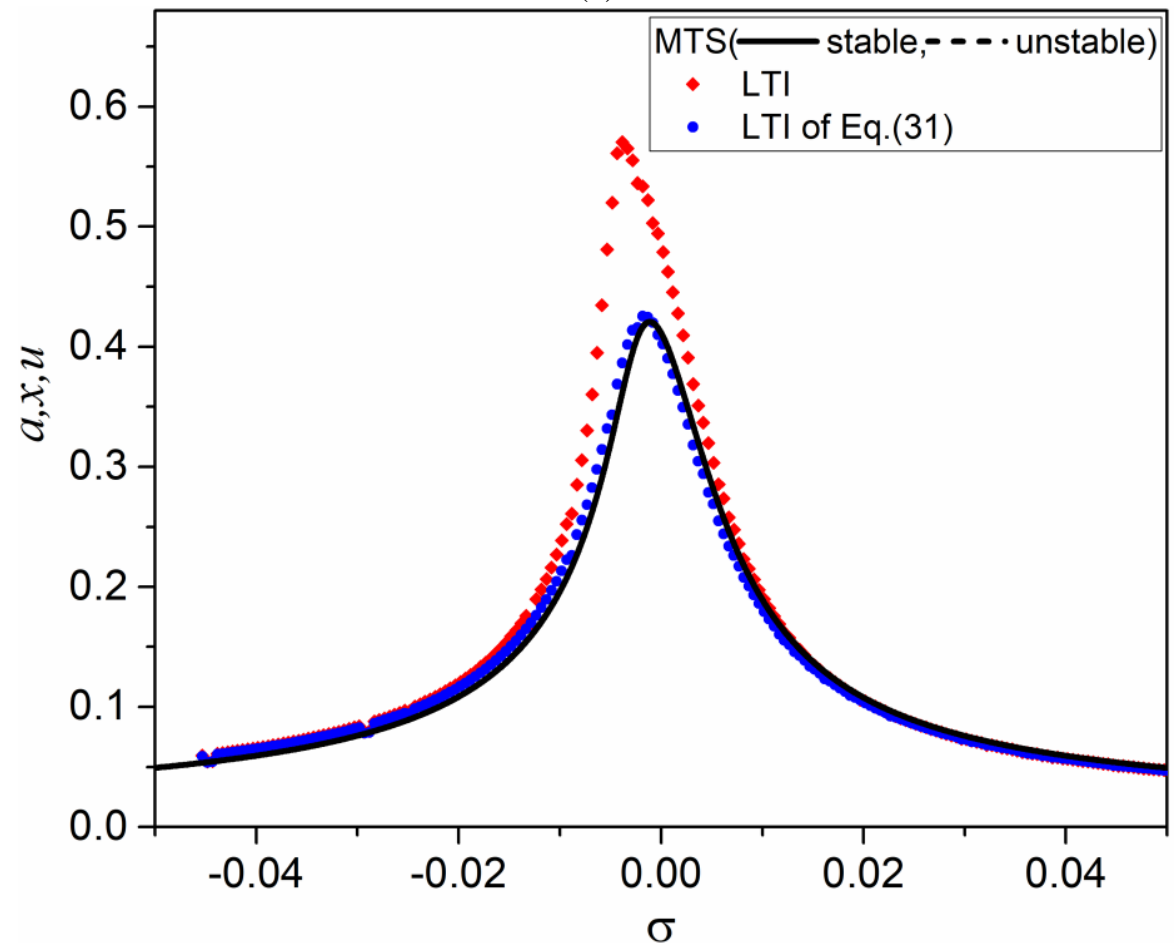

(c)

Fig. 2 Analytical and numerical frequency-response curves of the resonator against the frequency detuning parameter $\sigma$ for (a) $V_{D C}=10 \mathrm{~V}, V_{A C}=10 \mathrm{~V}$ and $\mu=0.01$, (b) $V_{D C}=15 \mathrm{~V}, V_{A C}=5 \mathrm{~V}$ and $\mu=0.01$, (c) $V_{D C}=1 \mathrm{~V}$, and $V_{A C}=15 \mathrm{~V}$ and $\mu=0.01$. The detuning parameter here is defined as $\Omega=\frac{1}{2} \omega_{n}+\sigma$. Note here that the frequency response 
equations obtained from MTS give only the dynamic solution, and hence the static solution is superimposed afterwards to compare to the LTI results.



(a)

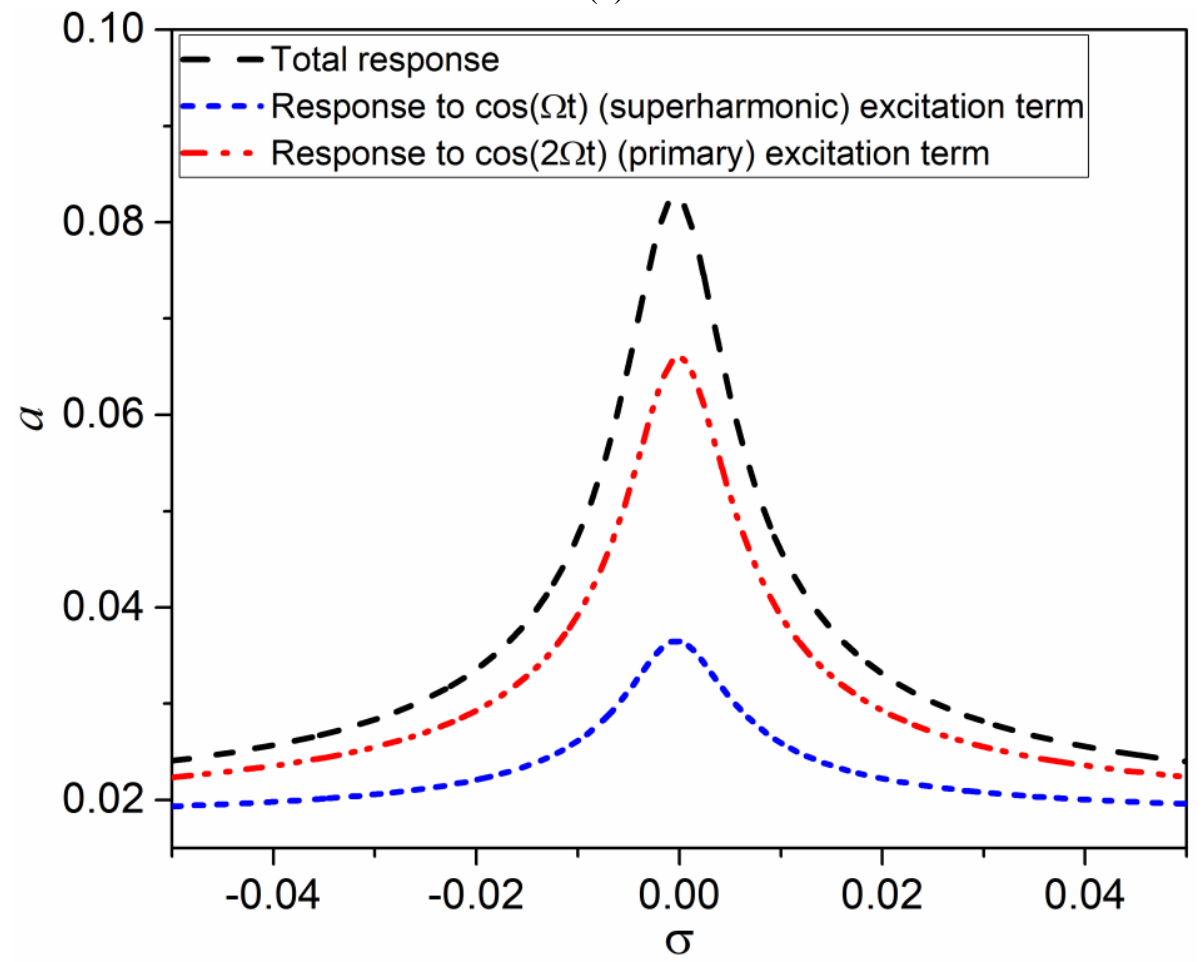

(b) 


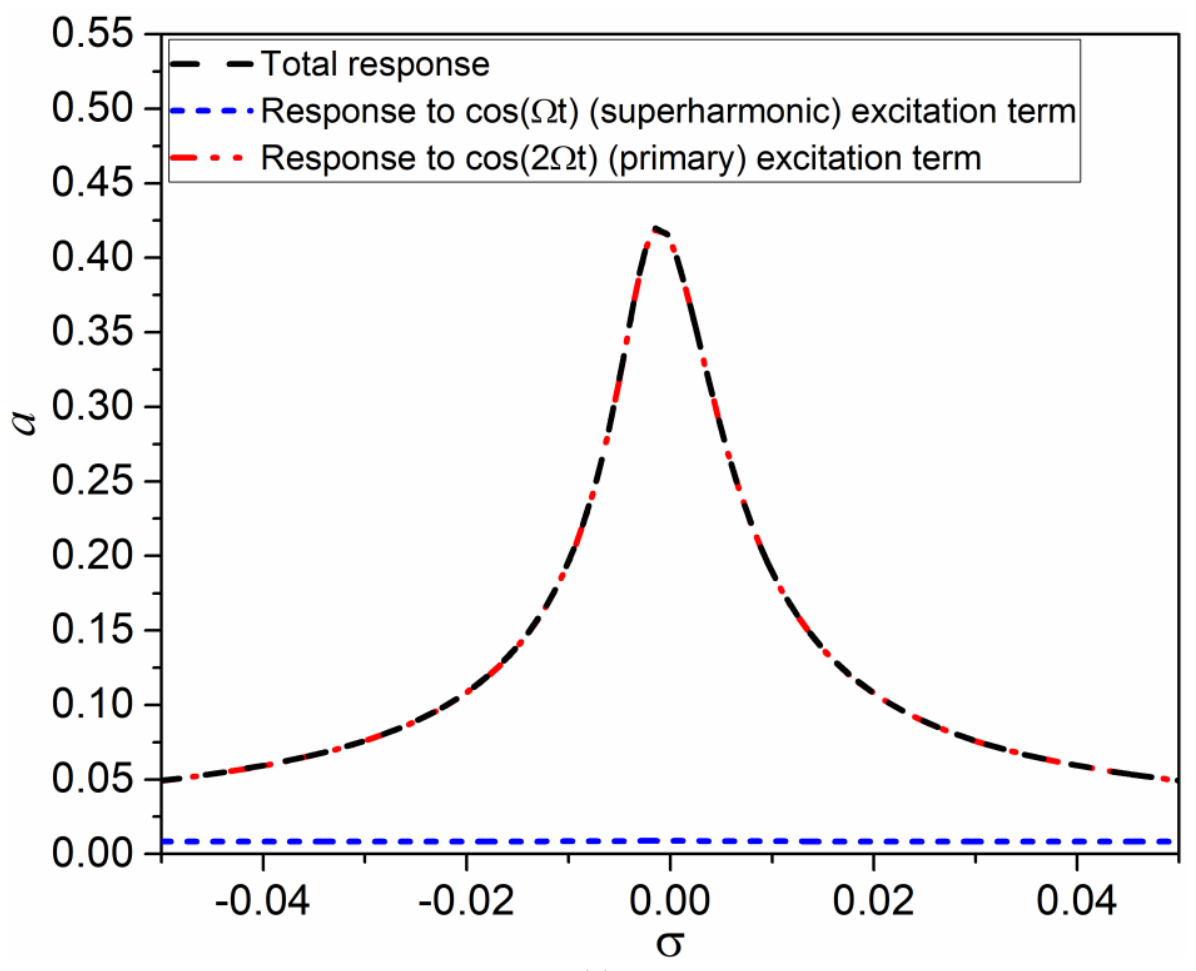

(c)

Fig. 3 Analytical frequency-response curves of the resonator against the frequency detuning parameter $\sigma$ for (a) $V_{D C}=10 \mathrm{~V}, V_{A C}=10 \mathrm{~V}$ and $\mu=0.01$, (b) $V_{D C}=15 \mathrm{~V}, V_{A C}=5 \mathrm{~V}$ and $\mu=0.01$, (c) $V_{D C}=1 \mathrm{~V}$, and $V_{A C}=15 \mathrm{~V}$ and $\mu=0.01$, showing the contribution from primary and superharmonic components to the overall resonators response. The detuning parameter here is defined as $\sigma=\Omega-\frac{1}{2} \omega_{n}$. The response shown here comprises of the dynamic solution obtained visa MTS superimposed onto the static solution. 